\title{
Robust transmission of Prioritized H.264/AVC Video EMPLOYING Cross-Layer Unequal Error Protection
}

\author{
Mussawir Ahmad Hosany ${ }^{1}$, Sunil Kumar ${ }^{2}$, Santosh Nagaraj ${ }^{3}$ \\ ${ }^{I}$ (Electrical and Electronic Engineering Department, University of Mauritius, Mauritius, \\ ${ }^{2,3}$ (Electrical and Computer Engineering Department, San Diego State University, San Diego, California, USA
}

\begin{abstract}
In this paper, we discuss a cross-layer unequal error protection (UEP) scheme, by jointly using unequal-rate convolutional codes (i.e., RCPC codes) and asymmetric 8PSK modulation at the physical layer, for robust transmission of prioritized H.264/AVC video packets over error-prone channels. The H.264/AVC video packets are assigned different priorities based on their contributions to the video quality. To minimize the effect of transmission errors on the reconstructed video quality, the proposed UEP scheme provides higher protection to the most important video packets at the cost of the least important packets. It is shown that the proposed cross-layer UEP scheme employing prioritized RCPC and asymmetric 8PSK modulation outperforms the corresponding equal-rate RCPC code and symmetric 8PSK modulation schemes.
\end{abstract}

Keywords: robust video transmission, unequal error protection, convolutional codes, RCPC codes, hierarchical/ asymmetric modulation, cross-layer, H.264/AVC video coding.

\section{Introduction}

Multimedia applications, such as video streaming are growing rapidly. Lately the H.264/AVC video compression standard has become popular because it provides better compression efficiency than its predecessors such as MPEG-4 and thus makes efficient use of the available channel bandwidth [1,2]. Since the compressed video bitstream is very susceptible to channel errors, different types of error resiliency schemes have been proposed in H.264 to reduce the effect of transmission errors [2,3].

In order to minimize the effect of transmission errors on the reconstructed video quality, the unequal error protection (UEP) schemes have been used [4-7]. In UEP, the more important video data (e.g., picture header and motion vectors) is given higher protection at the cost of less important data (e.g., residual DCT coefficients of P or B frames). The video bitstream can be partitioned into segments of different priorities according to their relative impact on video quality. At the physical layer, UEP can be achieved by using the forward error correction codes (typically with convolutional codes) or hierarchical (asymmetric) modulation where each bit label position has a different level of protection resulting from judiciously chosen signal constellations.

In [8-11], the hierarchical modulation schemes were investigated for multimedia applications. Ramachandran et al. [12] designed a digital HDTV broadcast system using scalable discrete cosine transform based source coding and multi-resolution quadrature amplitude modulation (QAM) under a joint source-channel coding framework. Chang et al. [13] proposed a UEP scheme by matching a hierarchical QAM to H.264 packets for AWGN channels, where important data is matched to more protected bits in a layered codec. A matched UEP scheme using hierarchical modulation was also proposed for terrestrial video broadcasting in [14]. In [15], Jiang et al. described another matched UEP scheme where they combined Trellis Coded Modulation (TCM) and non-uniform QAM schemes for transmitting H.264/AVC data. In [16], Arslan et al. presented a source-channel coding scheme using hierarchical modulation for progressive transmission. In [17], our coauthors recently described a UEP scheme for H.264 video by using RCPC codes and hierarchical QAM.

To date, the joint design of Rate-Compatible Punctured Convolutional (RCPC) codes and asymmetric modulation has received little attention. In this paper, we propose a novel cross-layer UEP scheme wherein the prioritized H.264/AVC video packets are offered appropriate protection at the physical layer according to their priority, by jointly using the unequal rate RCPC codes and the asymmetric 8-PSK modulation. The proposed UEP scheme provides graceful degradation of reconstructed video data against channel errors.

This paper is organized as follows. Section 2 briefly introduces the H.264/AVC standard along with a packet priority assignment schemes. Section 3 outlines the concepts associated with the convolutional codes (RCPC) as well as the asymmetric 8-PSK modulation in UEP. In Section 4, we describe the processes involved in the design and implementation associated with the proposed scheme. Section 5 explains the experimental setup and presents the simulation results as well as discussions for various equal-rate and prioritized RCPC code designs with asymmetric 8 PSK modulation applied to the prioritized H.264/AVC video data. Finally, Section 6 concludes the paper. 


\section{H.264/Avc Video Standard And Prioritization}

The H.264/AVC standard [1,2] is composed of two layers, namely the Video Coding Layer (VCL) for video compression and Network Abstraction Layer (NAL) for transport of compressed video data over networks [1]. NAL works in two kinds of mode: Single Slice mode and Data Partitioning (DP) mode. When using the DP mode, H.264 puts all variable length codes with the same data type together in each frame as described below.

The H.264/AVC compressed video data can be organized in packets of varying importance, depending on the frame type and DP type. In this paper, we classify the H.264/AVC bitstream into three priorities as shown below. Here priority 1 represents the most important data, while priority 3 represents the least important data. This scheme was discussed in our earlier work [7]:

- $\quad$ Priority 1: IDR frame, DPA of I frame and DPB of I frame.

- $\quad$ Priority 2: DPA of P frame, DPA of B frame and DPB of P frame.

- $\quad$ Priority 3 Queue: DPC of P frame, DPB of B frame and DPC of B frame

Since the instantaneous decoder refresh (IDR) is the most important frame in H.264 bitstream and cannot be partitioned, the entire frame is assigned to priority 1. It should be noted that data packets from the lowest priority queue can be dropped if necessary. This mechanism will help in maintaining equal lengths of the three queues, as discussed in Section 4.1.

\section{Uep Convolutional Codes And Asymmetric 8-Psk Modulation}

Convolutional codes are widely deployed in communication systems to encode digital data before transmission over error-prone channels, such as in the GSM system, deep space and satellite communications. A $(n, k, m)$ convolutional encoder maps $k$ input bits to $n$ output bits giving rise to a code rate of $k / n$ coded bitstream. Here $m$ is the number of shift registers in the encoder. The $n$ encoder output bits in a given time unit depend not only on the $k$ input bits at that time but also on the $m$ previous input bits. At the receiver, the transmitted bitstream can be decoded using the Viterbi algorithm $[22,23]$ to recover the original data. The RCPC codes can achieve UEP by puncturing off different amounts of coded bits of the parent code. Many applications of RCPC codes have been proposed in recent years [23-25].

\section{Asymmetric 8PSK Modulation}

A general model of multi-resolution signaling (also known as embedded or non-uniform constellation model) can be found in [26]. In this paper, the non-uniform 8-PSK constellation, with $m=\log _{2} 8=3$ hierarchical levels is considered, as shown in Fig. 1. The actual symbols are represented by small circles and are Gray coded. The first level and the second level virtual signal points are represented by " $x$ " and "+", respectively. The phase offset angles for general non-uniform PSK constellation $\theta_{i}$ are simplified as follows [7, 27]:

$$
\theta_{i}=\frac{\pi}{2} \beta^{i}, i=1,2, \ldots, m-1
$$

where $m=\log _{2} M$ and $M$ is the alphabet size. The reason for fixing the ratio of the angles for any subsequent levels of the signal constellation hierarchy $\beta$ to a constant is to facilitate the phase offset optimization (i.e., only a single parameter needs to be optimized rather than manipulating $(m-1)$ variables [27]).

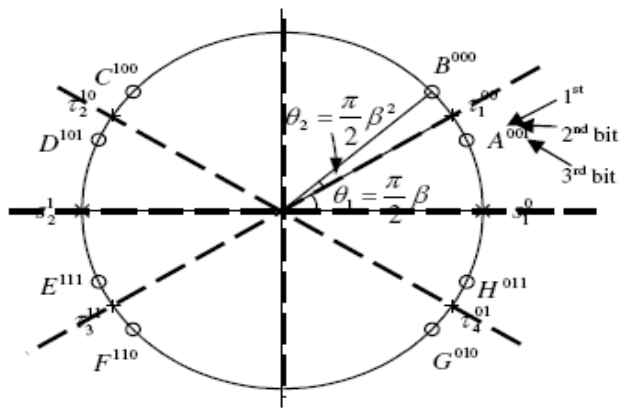

Fig. 1: Asymmetric 8 PSK constellation $[7,26]$.

When the phase offset ratio $\beta=0.5$, the system reverts to a uniform 8-PSK constellation. It was shown in [7] that the modulator achieves highest degree of unequal error protection among the three levels when $\beta$ ranges from 0.4 to 0.5 . Therefore, we have used the values of $\beta$ between 0.4 and 0.5 in our simulations in this paper. Also, the modulator is designed and implemented based on the mapping rules given in [7]. 


\section{Proposed System Design}

The design of the proposed cross-layer UEP scheme, employing the prioritized H.264/AVC video data at the application layer and equal-rate as well as prioritized (or unequal code rate) RCPC codes with asymmetric 8-PSK modulation at the physical layer, are explained in this section.

\subsection{Cross-layer UEP Scheme Equal-Rate RCPC Codes and Asymmetric 8PSK Modulation}

Fig. 2 shows the block diagram of our UEP scheme which uses convolutional encoder (i.e., equal-rate RCPC codes) and asymmetric 8PSK modulation. Fig. 2 shows the scheme in the form of a block diagram. We apply the equal rate RCPC code $\left(\mathrm{R}_{1}=\mathrm{R}_{2}=\mathrm{R}_{3}\right)$ to packets of each priority queue of H.264 video output. The outputs of the three codes are then multiplexed to form asymmetric 8PSK symbols by using 1 bit from each of the three priority queues to form a symbol. Here, the data from the highest (lowest) priority queue is assigned to the MSB (LSB) bit of the symbol, the middle priority data being assigned to the middle bit of the symbol. For a $512 \mathrm{kbps}$ video data, the length of each queue would be $r_{1}=r_{2}=r_{3}=512 / 3 \mathrm{kbps}$. By applying a $1 / 2$-rate RCPC code to every queue (i.e., $R_{1}=R_{2}=R_{3}=1 / 2$ ), the output data rate would be $1024 \mathrm{kbps}$.

Table 1 lists the different H.264 video bitrates, RCPC code rates and the output channel data rate. In all the cases, a mother code rate $1 / 2$ with puncturing periods of 8 and 6 has been used to generate RCPC code rates of $2 / 3,3 / 4$ and $4 / 5$ as suggested by Hagenauer [21] and Lee [22].

Table 1: H.264 Video Bit Rates, RCPC Code Parameters and Output Channel Data Rates

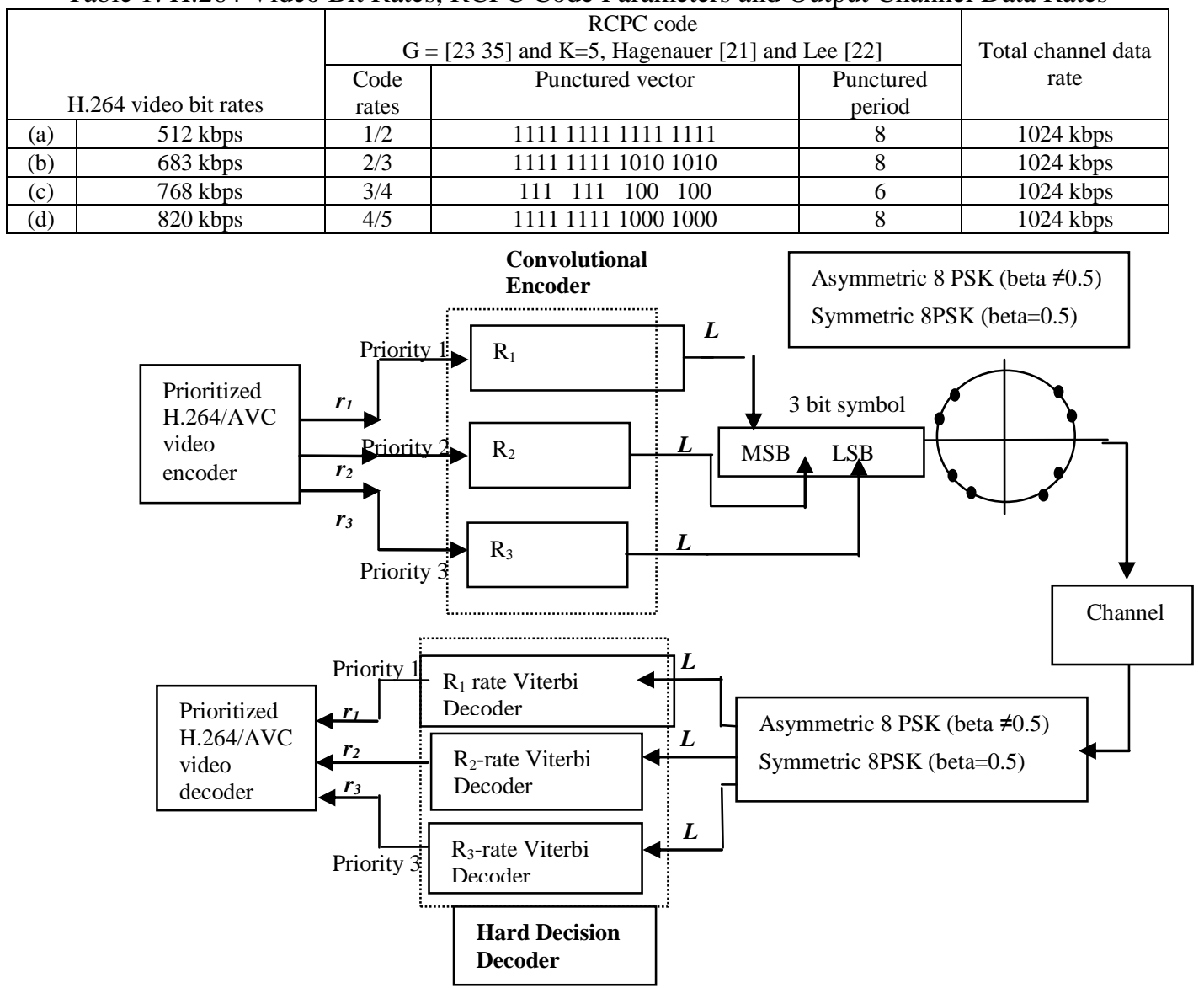

Fig. 2: Block diagram of the proposed cross-layer UEP scheme.

\subsection{Cross-layer UEP Scheme Employing Prioritized RCPC Codes and Asymmetric 8PSK Modulation}

The same design of Fig. 2 is used except that code rates $R_{1}, R_{2}$ and $R_{3}$ are unequal resulting in unequal video source rates $r_{1}, r_{2}$ and $r_{3}$. For example, in order to achieve an overall code rate of $1 / 2$, the three chosen RCPC codes in Table 2 are 8/18, 8/16, and $8 / 14$ with a puncturing period of 8 . 
Table 2: RCPC Code Parameters for Overall $1 / 2$-rate UEP Scheme

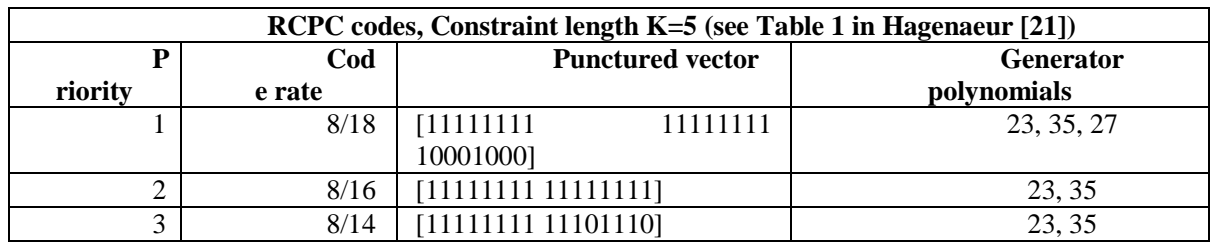

From Fig. 2, if the total source data rate is denoted as $R$, then according to the RCPC code rate arrangements, the percentage of data that should be fed to each priority can be found by using simple proportions as

$$
\begin{array}{ll}
r_{1}=\frac{R_{1}}{R_{1}+R_{2}+R_{3}} \times R & \underline{\text { or }} \\
r_{2}=\frac{\frac{8}{16}}{\frac{8}{18}+\frac{8}{16}+\frac{8}{14}} \times R=0.33 R & \text { (2) } \quad r_{3}=\frac{\frac{8}{18}}{\frac{8}{18}+\frac{8}{16}+\frac{8}{14}} \times R=0.29 R \\
&
\end{array}
$$

For a compressed video at $\mathrm{R}=512 \mathrm{kbps}$, the queue lengths of priority 1,2 and 3 can be obtained from Eqn. (1) to (3) as $29 \%, 33 \%$ and $38 \%$, respectively. For an overall code rate of $1 / 2$, the total channel data rate would be $1024 \mathrm{kbps}$.

Similarly, other overall 2/3,3/4 and 4/5 code rate cross-layer UEP schemes are designed. The RCPC code arrangements and source bitrate vary for each overall code rate, such that the total channel data rate is maintained at $1024 \mathrm{kbps}$ for a fair comparison among the UEP schemes. Table 3 shows the physical layer parameters used in the design of various overall code rate cross-layer UEP schemes. Different video bit rates are used and the amount of data in each queue, calculated from Eqn. (1) to (3), varies according to the RCPC code arrangements.

Table 3: Physical Layer Parameters for Overall Code Rates of 2/3, 3/4 and 4/5

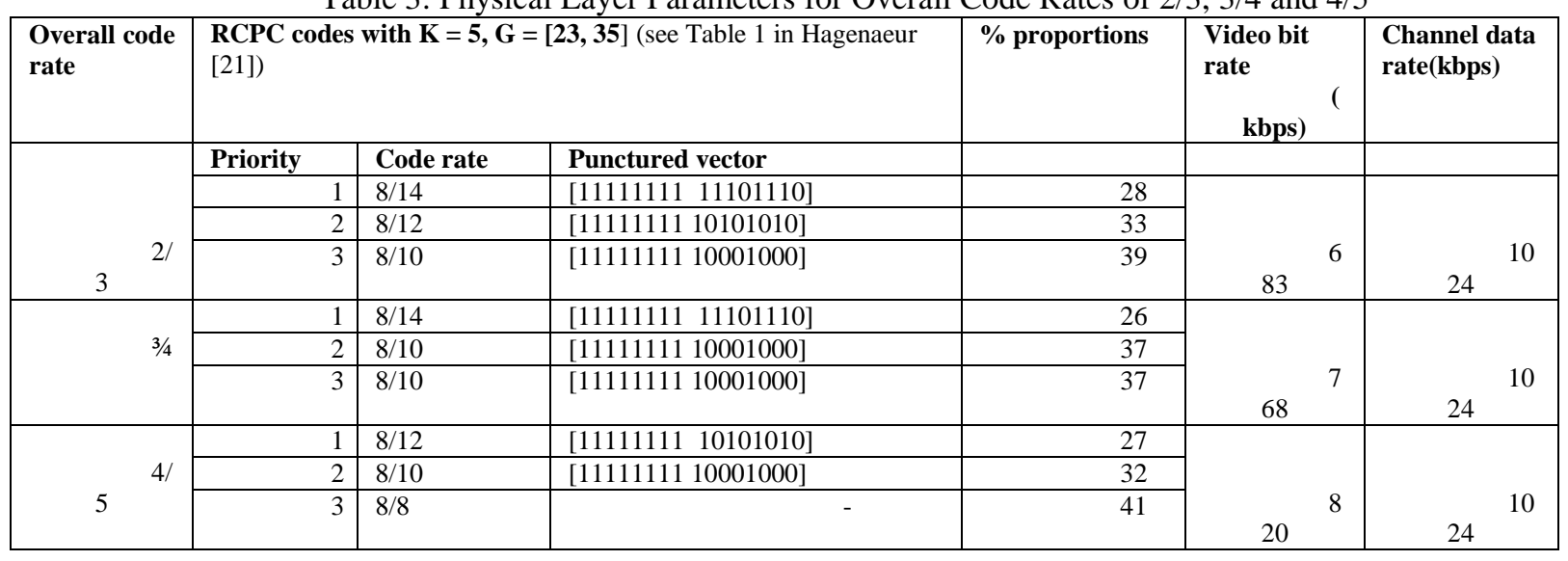

\section{Simulation Results And Discussions}

We have used the H.264/AVC Annex B (JM14.1) reference source code for encoding the CIF ( $352 \times 288$ pixels) Bus video test sequence at various bit rates mentioned in earlier sections. The video sequence was encoded at 30 frames/second for a GOP of 24 frames (IDR B P B P B P B P B P B I B...). The NAL size of 200 bytes was considered and a robust JM decoder with error concealment schemes was used. The AWGN channel model built in the MATLAB R2008a Simulink was used. The experiments were conducted by varying the CNR from $10 \mathrm{~dB}$ to $28 \mathrm{~dB}$ in steps of $2 \mathrm{~dB}$ and by varying the asymmetric 8-PSK modulation design parameter $\beta$ from 0.42 to 0.50 in steps of 0.02 . Each experiment was repeated 10 times with different random seeds and the obtained results were averaged.

Fig. 3 shows the video PSNR achieved by our UEP scheme for various $\beta$ values for different overall RCPC code rates. In Fig. 3(a), the asymmetric 8-PSK with $\beta$ value of 0.46 or 0.48 provide up to $0.6 \mathrm{~dB}$ PSNR gain compared to the symmetric case $(\beta=0.5)$ at a $\mathrm{CNR}$ of $18 \mathrm{~dB}$ (i.e., an $\mathrm{E}_{\mathrm{b}} / \mathrm{N}_{0}$ of about $13 \mathrm{~dB}$ ). For a high CNR of $28 \mathrm{~dB}$, the value of $\beta$ has no effect on the PSNR in Fig. 3(b) because the errors are very few. Note that rate $1 / 2$ RCPC code gives higher PSNR than the higher rate RCPC codes at lower CNR of $18 \mathrm{~dB}$, whereas it gives lower PSNR than the higher rate codes for high CNR of $28 \mathrm{~dB}$ when the channel errors are very few. 
Fig. 4 shows the performance of different overall RCPC code rates in our UEP scheme for various CNR values at $\beta=0.48$. At low CNR, the performance of our UEP scheme worsens as the overall code rate increases. For example, rate $1 / 2$ code has about $5 \mathrm{~dB}$ PSNR gain over the rate $2 / 3$ code at a CNR of $10 \mathrm{~dB}$, which reduces to about $2 \mathrm{~dB}$ at $\mathrm{CNR}$ of $18 \mathrm{~dB}$ and $21 \mathrm{~dB}$, respectively. As the CNR increases further, higher code rates outperform the code rate of $1 / 2$.

Performance of the equal-rate and prioritized RCPC codes in our UEP scheme is compared in Figs. 5 (a) and 5(b) for various overall RCPC code rates at $\beta=0.48$. The use of prioritized RCPC code outperforms the corresponding equal-rate RCPC codes for all the CNR values. For example, the UEP scheme based on overall 1/2-rate prioritized RCPC code achieves 3dB PSNR gain over the equal 1/2-rate RCPC code at a CNR of $18 \mathrm{~dB}$, because the former gives better protection to the higher priority packets as compared to the latter, which provides the same protection to the packets irrespective of their priority. Similarly, about $4 \mathrm{~dB}$ PSNR gain is observed for the UEP scheme based on overall 2/3-rate RCPC code at CNR of $18 \mathrm{~dB}$.

Fig. 6 shows two frames of the test video sequence for our UEP scheme. The UEP scheme employing prioritized RCPC code outperforms the corresponding equal-rate RCPC codes for $\beta$ at 0.48 .

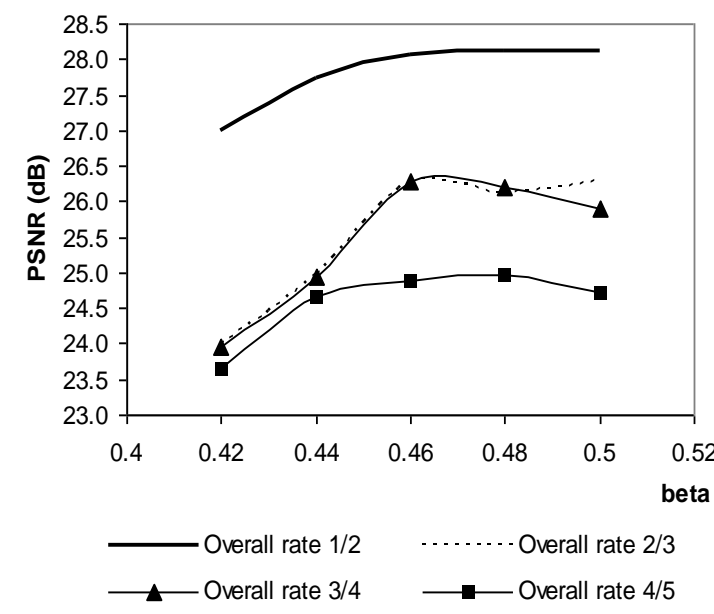

(a)

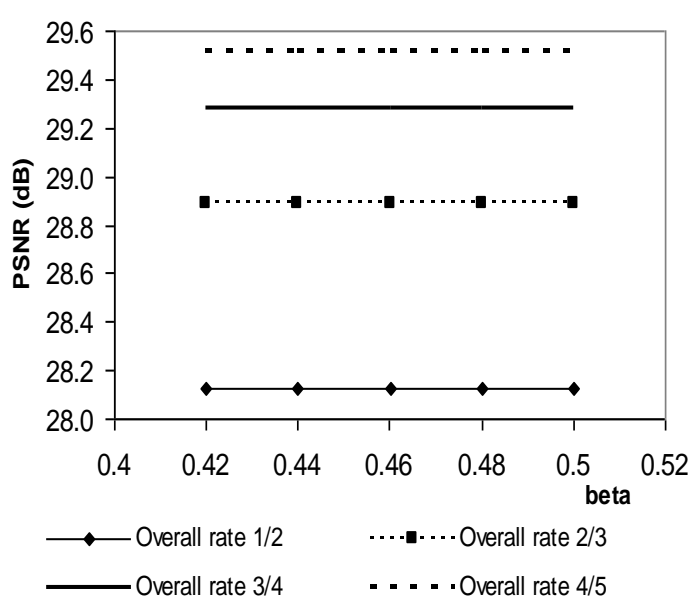

(b)

Fig. 3: PSNR curves for different $\beta$ values and overall RCPC code rates, at (a) $\mathrm{CNR}=18 \mathrm{~dB}$ and (b) $\mathrm{CNR}=28$ dB.

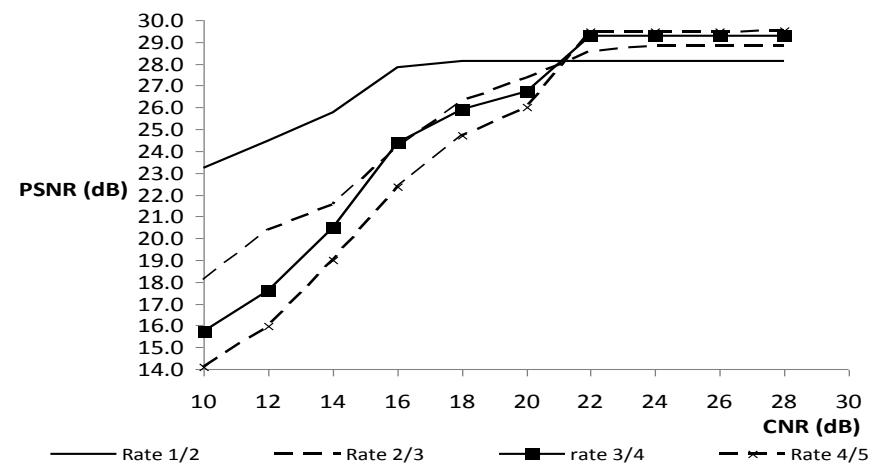

Fig. 4: PSNR performance of different RCPC codes rates for various CNR values.

\section{Conclusions}

In this paper, we discussed a cross-layer UEP scheme, by jointly using the prioritized (i.e., unequalrate) RCPC codes and asymmetric 8PSK modulation at the physical layer, for robust transmission of prioritized H.264/AVC video packets over error-prone wireless channels. It was shown through simulations that our UEP scheme performs better than the corresponding equal-code rate FEC and symmetric modulation schemes. We observed that the lower FEC code rates give better performance at lower CNRs, whereas the higher FEC code rates are better for higher CNRs. For the 1/2- rate RCPC based UEP scheme, there was only a small gain in PSNR for the asymmetric $(\beta<0.5)$ over the symmetric $(\beta=0.5)$ modulation scheme, because the $1 / 2$-rate RCPC code is powerful and hence the inherent UEP capability of the asymmetric modulator could not be utilized. 
However, for higher FEC code rates, our UEP scheme gives significant gains in PSNR with asymmetric modulation over the symmetric modulation. The reason for these gains is that the UEP capability of asymmetric

modulation is effectively utilized to protect more important video data when RCPC codes are not powerful enough.

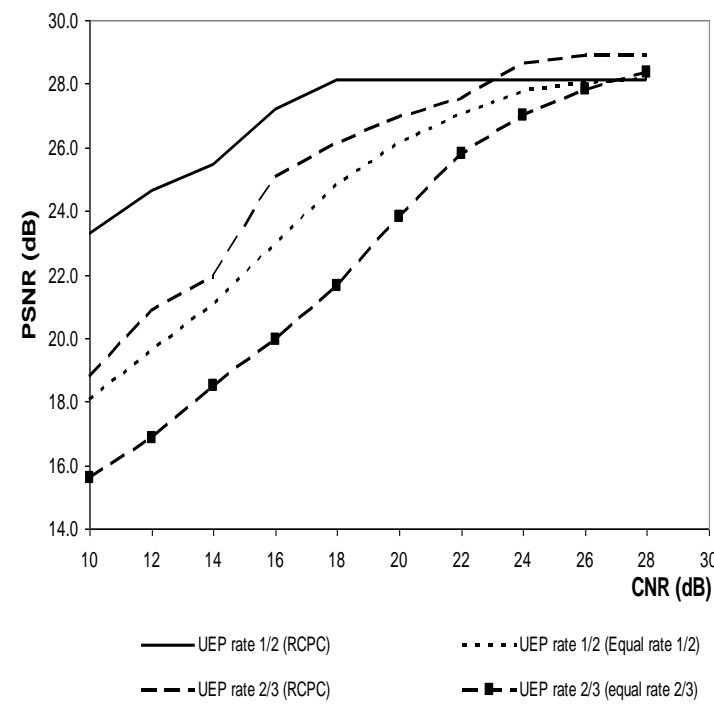

(a)

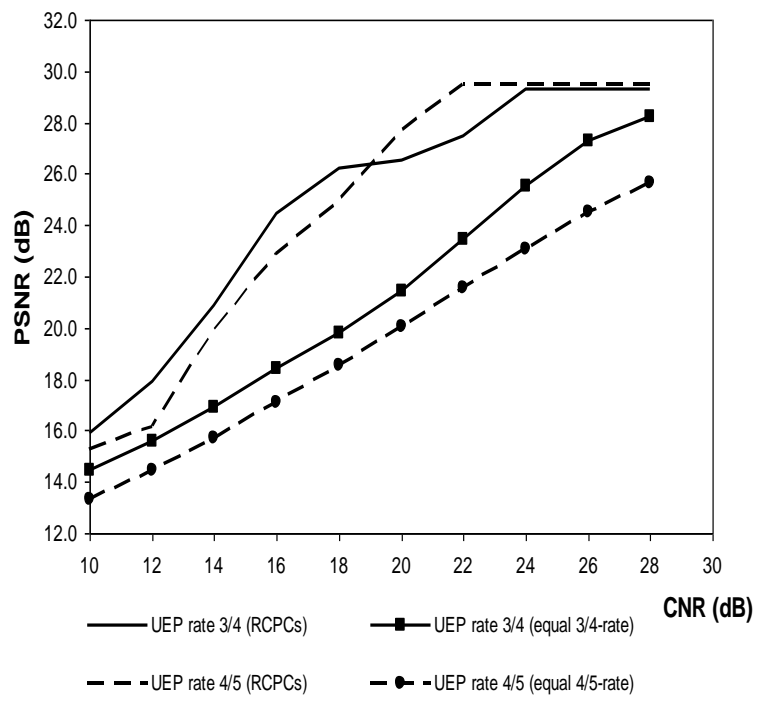

(b)

Fig. 5: Performance of the prioritized and equal-rate RCPC at various CNR values and $\beta=0.48$.

Frame No. 105

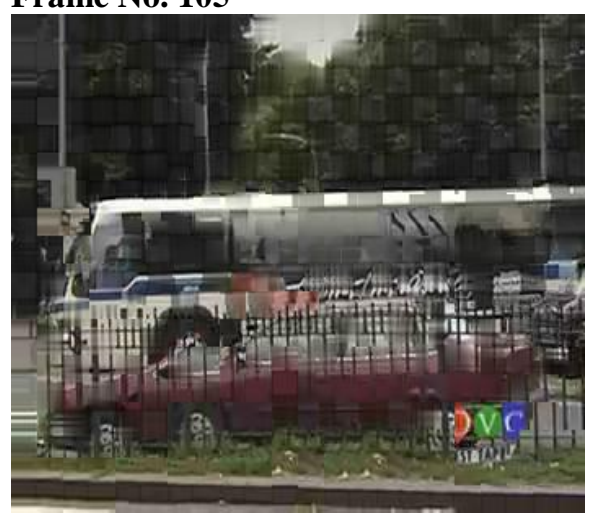

(c) Equal-rate RCPC rate $1 / 2$ scheme

PSNR 23.8 dB at CNR 18dB, beta $=0.48$
Frame No. 105

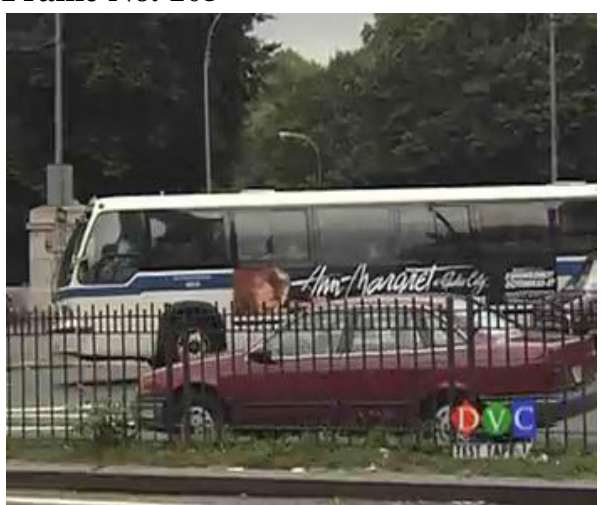

(d) Prioritized RCPC rate $1 / 2$ scheme PSNR $28.1 \mathrm{~dB}$, CNR $18 \mathrm{~dB}$, beta $=0.48$

Fig. 6: Test video frames for cross-layer overall code rate $1 / 2$ schemes.

\section{Acknowledgement}

The authors gratefully acknowledge the United States Department of State, Fulbright Commission for a grant to Dr. M.A. Hosany to carry out this research work at San Diego State University, San Diego, California, USA.

\section{References}

[1]. "Draft ITU-T recommendation and final draft international standard of joint video specification (ITU-T Rec. H.264/ISO/IEC 14 496-10 AVC," in Joint Video Team (JVT) of ISO/IEC MPEG and ITU-T VCEG, JVTG050r1, 2003

[2]. T. Wiegand, G. J. Sullivan, G. Bjøntegaard, and A. Luthra, "Overview of the H.264/AVC video coding standard," IEEE Trans. Cir. Syst. Video Technol., Vol. 13, pp. 560-576, July 2003.

[3]. S. Kumar, L. Xu, M. K. Mandal, and S. Panchanathan, "Error resiliency schemes in H.264/AVC video coding standard," Elsevier J.Visual Communication and Image Representation (Special issue: Emerging H.264/AVC Video Coding Standard), Vol. 17(2), pp. 425-450, April 2006.

[4]. L. F. Wei, "Coded modulation with unequal error protection," IEEE Trans. Commun., Vol. 41(10), pp. 1439-1449, Oct. 1993.

[5]. A. R. Calderbank and N. Seshadri, "Multilevel codes for unequal error protection," IEEE Trans. Inf. Theory, Vol. 39(7), pp. 12341248, July 1993. 
[6]. W. H. Heinzelman and R. Talluri, "Unequal error protection of MPEG-4 compressed video," Proc. International Conf. Image Processing, Vol. 2, pp. 530-534, 1999.

[7]. A. Bhatnagar, S. Kumar, A. Janarthanan, A. Annamalai Jr, M. Medley and J. Matyjas, "A multilevel unequal protection scheme for robust H.264/AVC video transmission over wireless channels," IEEE MILCOM, Boston, MA, USA, Oct. 2009.

[8]. M. B. Pursley and J. M. Shea, "Non-uniform phase-shift-key modulation for multimedia multicast transmission in mobile wireless networks," IEEE J. Sel. Areas. Commun., Vol. 17(5), pp. 774-783, May 1999.

[9]. M. B. Pursley and J. M. Shea, "Adaptive non-uniform phase-shift-key modulation for multimedia traffic in wireless networks," IEEE J. Sel. Areas. Commun., Vol. 18(6), pp. 1394-1407, Aug. 2000.

[10]. M. B. Pursley and J. M. Shea, "Multimedia multicast wireless communications with phase-shift-key modulation and convolutional coding," IEEE J. Sel. Areas. Commun., Vol. 17( 9), pp. 1999-2010, Nov. 1999.

[11]. J. Liu and A. Annamalai, "Multiresolution signaling for multimedia multicasting", Proc. IEEE Vehicular Technology Conference, Fall 2004, pp. 1088-1092.

[12]. K. Ramchandran, A. Ortega, K.M. Uz, and M. Vitterli, "Multiresolution broadcast for Digital HDTV using Joint Source/Channel Coding", IEEE J. Sel. Areas Communications, Vol. 11(1), 1993, pp. 6-23.

[13]. Y. C. Chang, S. W. Lee, and R. Komiya, "A low complexity unequal error protection of H.264/AVC video using adaptive hierarchical QAM," IEEE Trans. Consumer Electronics, Vol. 52(4), Nov 2006, pp. 1153-1158.

[14]. "Digital video broadcasting (DVB): Framing structure, channel coding and modulation for terrestrial television", European Standard 300744 V1.5.1, European Telecommunications Standard Institute (ETSI), Nov 2004.

[15]. S. Jiang, H. Lin, and S. K Tsou,"A new unequal error protection scheme for wireless multimedia transmissions", IEEE Region 10 Conference (TENCON 2007), Oct. 30 2007, pp. 1-4.

[16]. S. Arslan, P. Cosman, and L.B. Milstein, "Progressive source transmission using joint source-channel coding and hierarchical modulation in packetized networks," IEEE Globecom, 2009.

[17]. W.-H. Chung, S. Kumar, S. Paluri, S. Nagaraj, A. Annamalai, and J. Matyjas, "A cross-layer unequal error protection scheme for prioritized H.264 video using RCPC Codes and hierarchical QAM,” J. Information Processing Systems, 2012, to appear.

[18]. B. Sklar, Digital Communications - Fundamentals and Applications, Prentice Hall PTR, 2001.

[19]. S. Lin S and D. Costello, Error Control Coding: Fundamentals and Applications, Prentice-Hall, Englewood Cliffs, N.J., 1983.

[20]. A. Viterbi, "Convolutional codes and their performance in communication systems," IEEE Trans. Commun., Vol. 19(5), Part 1, pp. 751-772, Oct. 1971.

[21]. J. Hagenauer, "Rate-compatible punctured convolutional codes (RCPC codes) and their applications," IEEE Trans. Commun., Vol. 36(4), pp. 389-400, 1988.

[22]. L. H. C. Lee, "New rate-compatible punctured convolutional codes for Viterbi decoding," IEEE Trans. Commun., Vol. 42(12), pp. 3073-3079, 1994.

[23]. Y. Lee, S. Choi, and K. Kim, "Performance of RCPC codes with soft-decision decoding over Nakagami -m fading channels", IEEE Commun. Letts., Vol. 6, pp. 31-33, Jan. 2002.

[24]. A. Roongta and J. M. Shea, "Reliability based hybrid ARQ and rate-compatible punctured convolutional (RCPC) codes", Proc. IEEE Wireless Commun. and Networking Conf., Vol. 4, pp. 2105-2109, 21-25 March 2004.

[25]. H. Kim and G. L. Stuber, "Rate-compatible punctured SCCC codes," Proc. Vehicular Techol. Conf., Vol. 4, pp. 2399-2403, Oct. 2001.

[26]. P. Vitthaladevuni and M. Alouini, "Exact BER computation of generalized hierarchical PSK constellations," IEEE Trans. Commun., Vol. 51, pp. 2030-2037, Dec. 2003.

[27]. J. Lu, A. Annamalai Jr., and D. R. Vaman, "Reducing signal distortion due to transmission error via multi-resolution digital modulations", Wireless Telecommunications Symposium, April 2008, pp 360- 369. 